\title{
The Modulatory Effect of the Hypothalamus on Glucagon and Insulin Secretion in the Rat
}

\author{
A. B. Steffens \\ Department of Zoology, State University of Groningen, Haren (Gr.), The Netherlands
}

\begin{abstract}
Summary. Rats were provided both with brain cannulas, to permit infusion of fluids in the ventromedial and lateral hypothalamic areas (resp. VMH and LH), and with two heart catheters. In this way infusions of fluids and withdrawal of blood could be done in unanesthetized free moving animals. Infusion of norepinephrine (NE) in the VMH elicited glucagon release during the whole period of $\mathrm{NE}$ infusion whereas insulin levels did not change. This glucagon release could not be suppressed by $\alpha$ - and $\beta$-receptor blockade but it was suppressed by hexamethonium, a blocking agent of signal transmission in peripheral ganglia of the autonomic nervous system. On the other hand, infusion of $\mathrm{NE}$ in the LH elicited insulin release during the whole period of $\mathrm{NE}$ infusion whereas glucagon levels remained unchanged. It is argued that [1] the sympathetic nervous system is involved in glucagon release, [2] no $\alpha$ - and $\beta$-receptor mechanisms are involved during this glucagon release, [3] the parasympathetic nervous system is involved in insulin release during noradrenergic $\mathrm{LH}$ stimulation.
\end{abstract}

Key words: Ventromedial hypothalamus, lateral hypothalamus, insulin release, glucagon release, sympathetic nervous system, parasympathetic nervous system, blood glucose regulation

A large body of evidence has accumulated indicating that the nervous system plays an important role in the regulation of the release of insulin and glucagon from the endocrine pancreas [17]. Electrical stimulation of the vagal branches to the pancreas elicites insulin release [9]. Electrical stimulation of the splanchnic nerve, on the other hand, causes glucagon release and suppression of insulin release [1]. In addition to electrical stimulation, neurotransmitters involved in the action of the autonomic nervous system are capable of influencing the islets of Langerhans. Norepinephrine (NE), the sympathetic neurotransmitter, influences insulin release via stimulation of the $\alpha$ - and $\beta$-receptors of the B-cell of the islets of Langerhans. Stimulation of the $\alpha$-receptors inhibits insulin release while the $\beta$-receptors stimulate insulin release [11]. The $\mathrm{B}$-cell is influenced by circulating $\mathrm{NE}$ as well as NE released at nerve endings which are in contact with these cells [2]. On the other hand, both in vivo and in vitro studies show that acetylcholine, the parasympathetic neurotransmitter, has only a stimulatory effect on insulin release [12]. Furthermore, electrical stimulation of the vagus nerve results in a increase of insulin release which is suppressed by the anticholinergic drug atropine.

Though much is known about effects of peripheral activation of the autonomic nervous system on the islets of Langerhans, our knowledge about central mechanisms involved in this activation is rather limited. In a previous publication it was shown that injection of $0.1 \mu \mathrm{g}$ of NE bilaterally into the ventromedial hypothalamus (VMH) in rats elicited an immediate increase in both glucagon level and insulin level. Both levels reached a peak value 3 min after NE injection into the VMH and declined thereafter to pre-injection levels within two minutes. These reactions could not be abolished by atropine injection into the rats before $\mathrm{NE}$ injection into the $\mathrm{VMH}$, indicating that a muscarinic pathway is not responsible for the observed phenomenon [4]. On the other hand, injection of $0.1 \mu \mathrm{g}$ of NE bilaterally into the lateral hypothalamus (LH) elicited only an immediate rise in insulin which reached a peak $3 \mathrm{~min}$ after NE injection and declined thereafter to preinjection levels within two minutes. No changes in 
glucagon levels could be observed [4]. This reaction of insulin could be abolished by atropine given to the rats before $\mathrm{NE}$ injection into the $\mathrm{LH}$, indicating that a muscarinic pathway is probably involved in this reaction.

Since it has been demonstrated that injections of $\mathrm{NE}$ into the $\mathrm{VMH}$ and $\mathrm{LH}$ can elicit glucagon and insulin release from the islet of Langerhans, it is worthwhile to investigate whether infusions of NE in the $\mathrm{VMH}$ and $\mathrm{LH}$ can increase glucagon and insulin levels during the whole period that the NE infusion lasts. If, indeed, these reactions are elicited by NE infusions in the VMH and $\mathrm{LH}$, the question arises whether sympathetic and parasympathetic pathways are involved. To investigate the contribution of the sympathetic system, a peripheral blockade was given with (A) a combination of the $\alpha$ blocker phentolamine and the $\beta$ blocker propranolol to block peripheral sympathetic effects and (B) hexamethonium, a compound which suppresses the peripheral ganglia of the autonomic nervous system. To reveal the contribution of the parasympathetic system, a peripheral blockade with atropine was applied.

\section{General Methods}

\section{Subjects and Maintenance}

The animals in all experiments were male Wistar rats ranging in weight between $300-350 \mathrm{~g}$ at the beginning of the experiments. They were kept in individual plexiglass cages $(25 \times 25 \times 30 \mathrm{~cm})$ at room temperature $\left(20^{\circ} \mathrm{C} \pm 2^{\circ}\right)$ and had continuous access to food and water, unless otherwise stated. They were maintained on a 12-hr light-dark cycle, 6:00 a. m. $-6: 00 \mathrm{p}$. m. light). The standard C-rich diet (Muracon ${ }^{\ominus}$ Lab chow) contained $20 \%$ protein, $53.5 \%$ carbohydrate, $4.5 \%$ fat and $22 \%$ water with added vitamins and minerals. The rats were handled and weighed every morning at about 9:00 A.M.

\section{Surgery and Control of Body Weight}

All surgery was performed under ether anesthesia. A 1 to 2-week recovery period was allowed between surgeries. The experiments started as soon as the rats returned to pre-operative weight. There were no significant differences in body weight of the rats at the beginning of an experiment.

\section{Implantation of Brain Cannulas}

Permanent stainless steel cannula (L $21.0 \mathrm{~mm}$, O. D. $0.3 \mathrm{~mm}, \mathrm{I}$. D. $0.1 \mathrm{~mm}$ ) for chemical stimulation were stereotactically implanted bilaterally into either the VMH (De Groot co-ordinates AP: $+5.9, \mathrm{~V}:-2.7, \mathrm{~L}: \pm 0.7 \mathrm{~mm}$ ) or the LH (De Groot co-ordinates AP: $+5.4, \mathrm{~V}:-2.3, \mathrm{~L}: \pm 1.7 \mathrm{~mm}$ ) [3]. A sterile stainless steel obturator (L $23 \mathrm{~mm}$, O.D. $0.08 \mathrm{~mm}$ ) flush with the tip of the cannula was used between experiments to ensure that the cannula remained open and pyrogen free. The cannula was protected with a 21-g needle except for the last $4 \mathrm{~mm}$ at the top. The protective sleeve was affixed to the skull with acrylic. Sterile polyethylene tubing (L $7.0 \mathrm{~mm}$, O. D. $0.61 \mathrm{~mm}$, I. D. $0.20 \mathrm{~mm}$ ) was put around the cannula and obturator. A polyethylene cap ( $\mathrm{L} 6.0 \mathrm{~mm}$ ) was placed at the protruding end of the $21-\mathrm{g}$ needle to cover the free end of the cannula.

\section{Implantation of Heart Catheters}

All animals were provided with a heart catheter according to the technique described earlier [14]. This method allows blood sampling in unanesthetized, undisturbed, free moving rats. Whenever intravenous infusions were given, the rats were provided with a second heart catheter implanted in the contralateral (left) jugular vein. This smaller second catheter (O. D. $0.64 \mathrm{~mm}$, I. D. $0.28 \mathrm{~mm}$ ) permits blood flow from the acromiodeltoid-cephalic and anterior jugular vein to the external jugular vein. In general, rats did not lose weight after implantation of the second catheter, indicating that no serious blockade in venous return from the head occurred after surgery.

\section{Blood Sampling Procedure}

All experiments were performed between 11:00 a. m. and 2:00 p. m.; i. e., during the rat's light cycle. Food was removed $2 \mathrm{hr}$ before the start of the experiment. Blood samples of 0.06 (glucose only) or $0.3 \mathrm{ml}$ were taken for determination of blood glucose, plasma insulin and glucagon. The sampling schedule is stated separately for each experiment. A transfusion of citrated blood obtained by means of a heart puncture from a donor rat was given after each $0.3 \mathrm{ml}$ sample unless otherwise stated.

\section{VMH and LH Infusion Procedure}

After removal of the cap and the obturator, a sterile polyethylene tube (L $400 \mathrm{~mm}$, O. D. $0.61 \mathrm{~mm}$, I. D. $0.20 \mathrm{~mm}$ ) was connected to each cannula. The tube was filled with sterile test fluid except for the last $2.0 \mathrm{~cm}$ at the tip which contained sterile saline. A small air bubble of (around $0.06 \mu \mathrm{l}$ ) separated the two solutions. The far end of the tube was closed with a $30-\mathrm{g}$ plug to prevent any unwanted leakage into the brain. The animals were returned to their cages 30 minutes prior to the start of the experiment. The far ends of all tubing remained outside the cage so as not to disturb the animal [16]. At the start of the infusion, the plugs were removed and the tubing was quickly connected to the infusion pump. The infusion schedule is stated separately for each experiment. The disappearance of the small air bubble is designated as the start of either the VMH or the LH infusion. The duration of all VMH and LH infusions was $20 \mathrm{~min}(0.25 \mu \mathrm{l} / \mathrm{min})$. A constantly moving, second air bubble at the other end of the tube ensured uniform infusion rate.

NE solutions were diluted with sterile saline from a stock solution of $2 \mathrm{mg} / \mathrm{ml}$ solvent.

\section{Chemical Determinations}

The blood samples were transferred immediately to chilled $\left(0^{\circ} \mathrm{C}\right)$ centrifuge tubes, containing $5 \mu \mathrm{l}$ heparin solution $(500 \mathrm{U} / \mathrm{ml})$ as an anticoagulant and $5 \mu \mathrm{l}$ aprotinin solution $(1200 \mathrm{KIU} / \mu \mathrm{l}$, NOVO) to prevent proteolysis of glucagon. Blood glucose was measured by the ferricyanide method of Hoffman (Technicon Autoanalyzer TM II) with $0.05 \mathrm{ml}$ blood taken from the $0.3 \mathrm{ml}$ sample. The remaining $0.25 \mathrm{ml}$ blood was centrifuged at $4{ }^{\circ} \mathrm{C}$ and the supernatant stored at $-30^{\circ} \mathrm{C}$. Rat plasma immunoreactive insulin (IRI) and plasma immunoreactive glucagon (IRG) were determined by means of a radioimmunoassay kit (NOVO). Guinea pig serum M8309 served as antiserum for the insulin assay. Antiserum K964 was used for the glucagon assay and has very little cross reaction with gut glucagon. Duplicate assays were performed on $25 \mu \mathrm{l}$ 
plasma samples. Both in the insulin and glucagon assay, the bound and ${ }^{125}$ I-labeled hormone was separated by means of a polyethylene glycol solution $(23.75 \% \mathrm{wt} / \mathrm{wt})$ as suggested by Henquin et al. [8].

\section{Histology}

Cannula placement was investigated. The rats were anesthetized with ether and perfused with $10 \%$ formalin. The brains were removed and stored overnight in $10 \%$ formalin and $4 \%$ glucose. The brains were frozen quickly in melting isopentane $\left(-80^{\circ} \mathrm{C}\right)$ and cut at 40 microns. Brain slices were stained with cresyl fast violet, examined under a light microscope and compared with sections depicted in the atlas of De Groot [3].

\section{Experiment 1}

The purpose of this experiment was to investigate the plasma insulin, glucagon and blood glucose levels during infusion of $\mathrm{NE}$ at a rate of $12.5 \mathrm{ng} / \mathrm{min}$ in the VMH.

Method. Seven rats with an initial mean body weight of $300 \mathrm{~g}$ were used in these experiments. The rats randomly received two treatments, i. e., infusion of NE or NE-solvent. NE was administered at a dose of $12.5 \mathrm{ng} / \mathrm{min}$. The NE or its solvent was given over a $20 \mathrm{~min}$ period. The total volume infused was $5 \mu \mathrm{l}$ at a rate of $0.25 \mu \mathrm{l} / \mathrm{min}$. Each rat received the two infusions with an interval of at least one day. The order of the infusions was randomized for each animal. Blood samples were taken at $\mathrm{t}=-11,-6$ (blood glucose only), $-1,+1,+3,+5,+7,+9,+14,+19,+24$ and +29 min. NE infusion started at $t=0$.

Results. The results are presented in Figure 1. Infusion of NE resulted in a rise in glucagon and glucose levels when compared to the infusion of NE-solvent alone, though infusion of solvent caused a small increase in glucose levels. Insulin levels were not much influenced by NE infusion in the VMH. Solvent infusion there caused a small decrease in insulin levels.

\section{Experiment 2}

In the previous experiment it was shown that $\mathrm{NE}$ infusion in the VMH resulted in an increase of plasma glucagon and blood glucose. The aim of this study was to investigate whether the increase of plasma glucagon and blood glucose elicited by NE infusion in the $\mathrm{VMH}$ could be suppressed by administration of phentolamine and propranolol which induce $\alpha$ - and $\beta$-receptor blockade at the sympathetic effector.

Method. One week after termination of Experiment 1, the same seven rats received a systemic infusion of phentolamine $(10 \mu \mathrm{g} /$ $\mathrm{min})$ and propranolol ( $16 \mu \mathrm{g} / \mathrm{min}$, dissolved in saline) at a rate of $0.1 \mathrm{ml} / \mathrm{min}$. These doses of phentolamine and propranolol were chosen because the dose of phentolamine can abolish a NE induced suppression of insulin release whereas the dose of propranolol can prevent a rise in insulin after administration of the $\beta$ receptor stimulant isoprenaline (J.H. Strubbe, personal communication). The infusion of phentolamine and propranolol started 35 min before infusion of NE in the VMH. NE was administered at a dose of $12.5 \mathrm{ng} / \mathrm{min}$ over a period of $20 \mathrm{~min}$. Blood samples were taken at $\mathrm{t}=-16,-6,0,+10,+20,+29,+31,+33,+35$, $+37,+39,+44,+49$, and $+59 \mathrm{~min}$. Systemic infusion of phentolamine and propranolol started at $t=-5$ and NE infusion in the VMH at $\mathrm{t}=+30$.

Results. The results are presented in Figure 2. In this figure the results of Experiment 2 are compared to the results of the NE infusion in the VMH of Experiment 1. During phentolamine and propranolol infusion no change in insulin or glucagon could be observed. However, a large decline in glucose levels occurred. After the start of the NE infusion in the $\mathrm{VMH}$, glucagon levels rose in the same way as in Experiment 1 in which phentolamine and propranolol were not administered. With respect to glucose, the same increase could be observed as in Experiment 1 though the increase started from a much lower basal glucose level. Insulin levels did not differ much between experiments.

\section{Experiment 3}

The aim of this experiment was to investigate whether hexamethonium could suppress the glucagon rise elicited by NE infusion in the VMH. Hexamethonium was chosen because it suppressed signal transmission in the peripheral ganglia of the sympathetic and parasympathetic system. Peripheral ganglion blockade is probably more appropriate to suppress the glucagon rise elicited by NE infusion in the VMH because (A) $\alpha$ - and $\beta$-receptor blockade could not suppress the above mentioned glucagon rise and (B) other mechanisms of signal transmission of the sympathetic system to the islets of Langerhans may occur besides an $\alpha$ - and $\beta$-receptor mechanism [10].

Method. One week after termination of Experiment 2, the same seven rats received a systemic infusion of hexamethonium $(30 \mu \mathrm{g} /$ $\mathrm{min}$, dissolved in saline) at a rate of $0.1 \mathrm{ml} / \mathrm{min}$. This dose was chosen because it could suppress free fatty acid release elicited by preganglionic stimulation of the sympathetic system [7]. The infusion of hexamethonium was started $16 \mathrm{~min}$ before infusion of $\mathrm{NE}$ in the VMH. NE was administered at a dose of $12.5 \mathrm{ng} / \mathrm{min}$ over a period of $20 \mathrm{~min}$. Blood samples were taken at $\mathrm{t}=-10,-1,+5$, $+10,+15,+17,+19,+21,+23,+25,+30,+35$, and +40 min. Systemic infusion of hexamethonium started at $t=0$ and $\mathrm{NE}$ infusion in the VMH at $\mathrm{t}=+16$.

Results. The results are presented in Figure 3. In this figure the results of Experiments 3 are compared to the results of the NE infusion in the VMH of Experiment 1. During hexamethonium infusion both insulin and glucagon declined whereas glucose remained nearly at the same level. After the start of the NE infusion in the VMH, the glucagon and insulin rose slightly. However, glucose increased in the same way as in Experiment 1. 

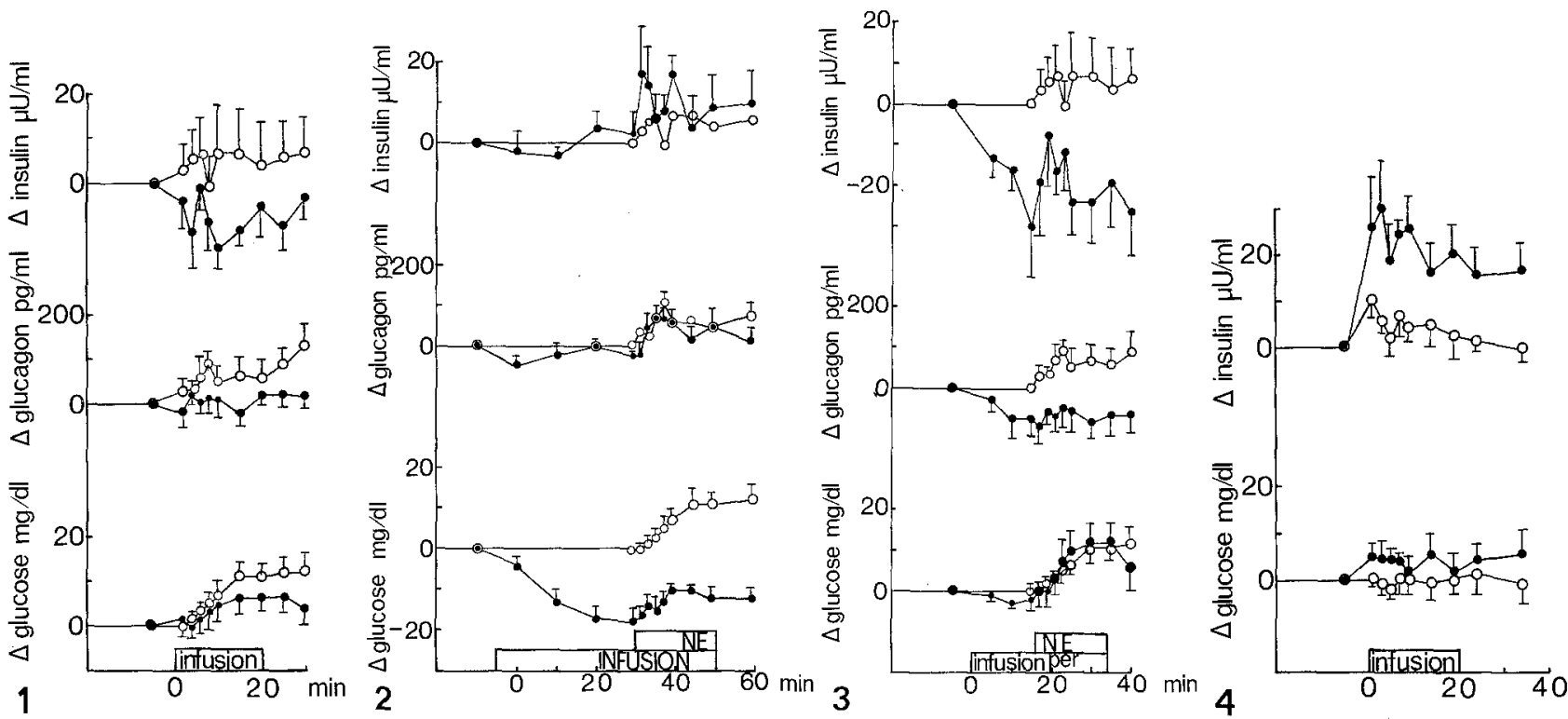

Fig. 1. Mean changes in plasma insulin, glucagon and blood glucose levels during infusion of either NE O_- O or NE-solvent VMH. The average of the levels at -11 and -1 was taken as the 0 level. Vertical bars mean SEM

Fig. 2. Mean changes in plasma insulin, glucagon and blood glucose levels during systemic infusion of phentolamine and propranolol and $\mathrm{NE}$ infusion in the VMH $\longrightarrow$ compared with those after NE infusion in the VMH without phentolamine and propranolol blockade $\mathrm{O}-\mathrm{O}$. The average of the levels at $\mathrm{t}=-16$ and $\mathrm{t}=-6$ was taken as the 0 level. Vertical bars mean SEM

Fig. 3. Mean changes in plasma insulin, glucagon and blood glucose levels during systemic infusion of hexamethonium and NE infusion in the VMH - compared with those after NE infusion in the VMH without hexamethonium blockade $\mathrm{O}-\mathrm{O}$. The average of the levels at $\mathrm{t}=-11$ and $\mathrm{t}=-1$ was taken as the 0 level. Vertical bars mean SEM

Fig. 4. Mean changes in plasma insulin and blood glucose levels during infusion of either NE - or NE-solvent $\mathrm{O}-\mathrm{O}$ in the $\mathrm{LH}$. The average of the levels at $\mathrm{t}=-11$ and $\mathrm{t}=-1$ was taken as the 0 level. Vertical bars mean SEM

\section{Experiment 4}

In the previous experiments it was shown that infusions of $\mathrm{NE}$ in the $\mathrm{VMH}$ elicited mainly glucagon release. Bcause in earlier work [4] it was observed that $\mathrm{NE}$ injection into the $\mathrm{LH}$ elicited an immediate insulin release of short duration (about $3 \mathrm{~min}$ ), the question arose whether infusion of NE in the LH over a period of $20 \mathrm{~min}$ could elicit a more prolonged rise in insulin level.

Method. Seven rats with an initial body weight of $300 \mathrm{~g}$ randomly received two treatments; i. e., infusion of either NE or NE-solvent. $\mathrm{NE}$ was adminstered at a dose of $12.5 \mathrm{ng} / \mathrm{cannula} / \mathrm{min}$. The NE or its solvent was given over a $20 \mathrm{~min}$ period with a total volume infused in the LH of $5 \mu \mathrm{l}$ at a rate of $0.25 \mu \mathrm{l} / \mathrm{min}$. Each rat received the two infusions randomly with an interval of at least one day between. Blood samples were taken at $\mathrm{t}=-11,-6$ (blood glucose only), $-1,+1,+3,+5,+7,+9,+14,+19,+24$, and +34 min. NE infusion in the LH started at $t=0$.

Results. The results are presented in Figure 4. Infusion of $\mathrm{NE}$ in the $\mathrm{LH}$ resulted in a rise in insulin levels when compared to the infusion of NE solvent alone. Glucose rose slightly after NE infusion in the $\mathrm{LH}$ as compared to NE solvent infusion.

\section{Discussion}

The main findings of these experiments are (A) infusion of NE in the VMH elicits glucagon release during the entire period of NE infusion (B), this glucagon release cannot be suppressed by $\alpha$ - and $\beta$-receptor blockade but it is suppressed by hexamethonium, a blocking agent of signal transmission in the peripheral ganglia of the autonomic system (C) infusion of NE in the LH elicits insulin release during the entire period of NE infusion.

The results of this study show that NE stimulation of the VMH elicits glucagon release with concomitant increase in glucose whereas insulin levels do not change much, indicating that the VMH can be considered as an area involved in regulation of glycogenolysis. This is in agreement with the work of Shimazu (for a review see [13]) who found that NE stimulation of the VMH elicited an immediate increase of active liver phophorylase which leads to the break-down of liver glycogen. Shimazu found that a direct sympathetic pathway to the liver elicited activation of liver phosphorylase. This direct sym- 
pathetic activation of the liver is supported by the concomitant increase in glucagon, also elicited by $\mathrm{NE}$ stimulation of the VMH. To determine whether the sympathetic system is involved in release of glucagon $\alpha$ - and $\beta$-receptor blockade was used. However, suppression could not be reached by means of this blockade. Two alternatives can explain this lack of suppression: (A) transmission of sympathetic activity to the A-cell of the islets of Langerhans is not by means of an $\alpha$ - or $\beta$-receptor mechanism, or (B) release of glucagon is not influenced by activity of the sympathetic system. To investigate the first alternative, a blockade of the sympathetic peripheral ganglia with hexamethonium was given. A suppression of VMHelicited glucagon release was observed. This means that if the sympathetic system is involved in $\mathrm{VMH}$ elicited glucagon release it must be by a mechanism other than $\alpha$ - and $\beta$-receptor activity. Recently it has been found that the C-terminal tetrapeptide amide of cholecystokinin is present in nerve terminals in the islets of Langerhans and that this tetrapeptide is a potent releaser of islet hormones [10]. This finding might explain the finding that $\alpha$ - and $\beta$-receptor blockade is without effect on VMH elicited glucagon release. However, it could be argued that VMH elicited glucagon release is caused by a muscarinic parasympathetic mechanism because hexamethonium also blocks the peripheral ganglion of the parasympathetic system. This argument cannot be valid because VMH elicited glucagon release is not suppressed by systemic atropinization [4].

Some other observed phenomena merit attention. A profound decline in glucose occurred in experiment 2 during systemic infusion of phentolamine and propranolol. This decline in glucose was not accompanied by changes of either insulin or glucagon, the most important hormones regulating glucose levels. To explain this decline of glucose, it might be that continuous $\alpha$ - and $\beta$-receptor activation of liver cells is responsible for a normal basal glucose level. When this activation is blocked by phentolamine and propranolol, a decline of glucose may ensue without effects on insulin and glucagon.

On the other hand, systemic infusion of hexamethonium caused a decrease in both insulin and glucagon levels without much change in glucose level (see Fig. 3). The decreases in insulin and glucagon can be explained by the suppression of autonomic activation of hormone release from the islets of Langerhans. There are many indications at the moment that the islet of Langerhans is under continuous control of the autonomic nervous system [11, 17]. NE infusion in the $\mathrm{LH}$ elicited insulin release during the whole infusion period (see Fig. 4). This observation is also in agreement with that of Shimazu
[13] who found that activation of the LH stimulated the conversion of inactive liver synthetase D into active liver synthetase I. Shimazu found that activation of liver synthetase was elicited by a direct parasympathetic pathway. The results of this investigation show that LH stimulated insulin release can contribute to activation of liver synthetase because insulin is a potent stimulator of conversion of liver synthetase D into liver synthetase I. Moreover, NE stimulation of the LH does not elicit glucagon release [5]. LH stimulated insulin release is mediated by the parasympathetic system because systemic atropinization or subdiaphragmatical vagotomy abolishes $\mathrm{LH}$ stimulated insulin release [15].

It can be concluded that (A) noradrenergic activation of the $\mathrm{VMH}$ and $\mathrm{LH}$, elicits glucagon and insulin release, (B) noradrenergic $\mathrm{VMH}$ stimulated glucagon release is probably mediated by the sympathetic nervous system, (C) $\alpha$ - and $\beta$-receptor mechanisms are not involved and (D) noradrenergic LH stimulated insulin release is probably mediated by the parasympathetic nervous system.

\section{References}

1. Bloom SR, Edwards AV, Vaughan NJA (1974) The role of the autonomic innervation in the control of glucagon release during hypoglycaemia in the calf. J Physiol (Lond) 236: 611-623

2. Cegrell L, Falck B, Hellman B (1964) Monoaminergic mechanisms in the endocrine pancreas. In: Brolin SE, Hellman $\mathrm{B}$, Knutsen $\mathrm{H}$ (eds) The structure and metabolism of the pancreatic islets. Pergamon, New York, pp 429-435

3. De Groot J (1959) The rat forebrain in stereotaxic coordinates. Verh Konink Ned Akad Wetenschap [Afdel Natuurkunde] Sect II: pp 1-40

4. De Jong A, Strubbe JH, Steffens AB (1977) Hypothalamic influence on insulin and glucagon release in the rat. Am $J$ Physiol 233: E380-E388

5. Flik G, Kuipers F, Lotter EC, Steffens AB (Submitted for publication) The modulatory effect of meal-related stimuli on centrally mediated insulin secretion. Endocrinology

6. Frohman LA, Bernardis LL (1971) Effect of hypothalamic stimulation on plasma glucose, insulin and glucagon levels. Am J Physiol 221: 1596-1603

7. Havel RJ, Goldfien A (1959) The role of the sympathetic nervous system in the metabolism of free fatty acids. J Lipid Res 1: 102-108

8. Henquin JC, Malvaux P, Lambert AE (1974) Glucagon immunoassay using polyethylene glycol to precipitate antibody bound hormone. Diabetologia 10: 61-68

9. Kaneto A, Kosaka K, Nakao K (1967) Effects of stimulation of the vagus nerve on insulin secretion. Endocrinology 80: $530-536$

10. Rehfeld JF, Larsson LI, Goltermann NR, Schwartz TW, Holst JJ, Jensen SL, Morley JS (1980) Neural regulation of pancreatic hormone secretion by the C-terminal tetrapeptide of CCK. Nature 284: 33-38

11. Robertson RP, Porte D (1973) Adrenergic modullation of basal insulin secretion in man. Diabetes 22: 1-8 
12. Sharp R, Culbert S, Cook J, Jennings A, Burr IM (1974) Cholinergic modification of glucose-induced biphasic insulin release in vitro. J Clin Invest 53: 710-716

13. Shimazu T (1979) Nervous control of peripheral metabolism. Acta Physiol Pol 30 [Suppl 18]: 1-18

14. Steffens AB (1969) A method for frequent sampling of blood and continuous infusion of fluids in the rat without disturbing the animal. Physiol Behav 4: 833-836

15. Steffens AB, Lotter EC (submitted for publication) Lateral hypothalamic and vagal control of the endocrine pancreas in the rat. Am J Physiol

16. Strubbe JH, Mein CG (1977) Increased feeding in response to bilateral injection of insulin antibodies in the VMH. Physiol Behav 19: 309-313

17. Woods SC, Porte D (1974) Neural control of the endocrine pancreas. Physiol Rev 54: 596-619

\author{
A. B. Steffens \\ Department of Zoology \\ State University of Groningen \\ NL-Haren (Gr) \\ The Netherlands
}

\section{Discussion after Steffens' Presentation}

Berthoud: Where did the histology reveal your LH cannulas to be?

Steffens: They were exactly centered in the LH area, which of course is very large.

Berthoud: Then you might expect a large spread of your administered drug?

Steffens: That's true, but not to the VMH since that would be expected to elicit an increase of glucagon secretion and it didn't occur.

Sclafani: Did you check your rats for NE-elicited eating?

Steffens: They did not have food available, but when the $\mathrm{NE}$ was added to the VMH, they appeared to want food. This was not true when the $\mathrm{LH}$ was stimulated.

Sclafani: How do your results relate to the cephalic release of insulin?

Steffens: I suspect that the cephalic response is due to increased nor-adrenergic tone in the $\mathrm{LH}$ and/or perhaps a decreased activity in the VMH. The net result would be an increase of insulin secretion in response to eating and would enable the animal to eat more food.

Smith: Why didn't insulin increase when you infused NE into the VMH? After all, glucose levels did increase and you did observe an increase of insulin when the NE was added acutely.

Steffens: It may be that the dose was too low because only $12.5 \mathrm{ng} \mathrm{NE} / \mathrm{min}$ was administered against $100 \mathrm{ng}$ in the experiment where NE was administered a cutely, or that the elevated glucagon somehow interfered with insulin secretion.

Smith: Were the total volumes and the potential spread of the drugs the same in the injection and the infusion experiments?

Steffens: In the infusion experiments, I gave $0.25 \mu \mathrm{l} / \mathrm{min}$ as opposed to $1 \mu \mathrm{l}$ over 2 or $3 \mathrm{sec}$ in the injection experiments. It's difficult to make easy comparisons between the two conditions.

Nicolaidis: In the experiment where insulin was increased and there was no change of glucagon, why was no secondary decrease of glucose caused by the insulin?
Steffens: I don't know; there must be some unknown competing mechanism.

Shimazu: We have recently put NE into the VMH of the unanesthetized and freely moving rabbit and also observed an increase of insulin and glucagon. However, both responses were relatively small. When we injected epinephrine into the $\mathrm{VMH}$ at the same molar dose $\left(5 \times 10^{-8} \mathrm{M}\right)$, both responses and especially the increase of glucagon were much larger. When we put the same amount of acetylcholine into the VMH, there was an increase of glucagon, a gradual increase of glucose and no change of insulin. This glucagon response could be blocked by the central administration of hexamethonium (Endocrinology, in press).

Steffens: We have made exactly the same observation with peripheral hexamethonium.

Shimazu: When epinephrine was put into the $\mathrm{LH}$, there was an increase of insulin with no change of glucagon. So in general, our results agree with yours.

Steffens: Yes, it's interesting that there are apparently two sets of receptors in the $\mathrm{LH}$ which can cause an increase of insulin secretion because injection of both $\mathrm{NE}$ and $\mathrm{ACh}$ elicit that reaction.

Berthoud: Do you think that if you infused glucose intravenously to prime the islets that central NE might cause a greater increase of insulin?

Steffens: That's possible. We haven't done that, but perhaps someone knows the answer.

Porte: If you give glucose to stimulate the islet, you'll get a quite marked potentiation of insulin secretion to other secretagogues. Likewise, if you prime the B-cells with other stimulants and then add glucose, you'll see a positive interaction (J Clin Endocrinol Metab (1979) 48: 946). The only time you won't see this interaction is when you prime first with glucose and then add more glucose as the stimulant.

Bray: Have you measured hypothalamic content or turnover of NE in these experiments?

Steffens: No, we haven't. 